\title{
The Importance of Having a Signature that is Difficult to Imitate or Forge
}

Andrea Thomas-Dobson

Forensic Document Examiner 38 Mount View, Sutton Coldfield, West Midlands, Birmingham, B757DT, UK

*Corresponding author: Forensic Document Examiner, 38 Mount View, Sutton Coldfield, West Midlands Birmingham, B757DT, UK, Tel: 07504603433; E-mail: thomasandrea1311@gmail.com

Received date: October 14, 2017; Accepted date: October 24, 2017; Published date: October 30, 2017

Copyright: () 2017Dobson AT. This is an open-access article distributed under the terms of the Creative Commons Attribution License, which permits unrestricted use, distribution, and reproduction in any medium, provided the original author and source are credited.

Keywords Signature; Forensic handwriting; Writing behavior; Direction of strokes

\section{Commentary}

Having examined and compared signatures for the past ten (10) years I have seen everything from legible to non-legible, complex signatures.

However I have one statement repeating itself: the importance of having a signature that is difficult to forge.

What does it mean to have a signature that is difficult to imitate/ forge? This article is intended to give suggestions to improve your signature so that it cannot be easily forged. Your signature identifies you.

Reed Hayes, in his book Forensic Handwriting Examination, states that a person's signature serves as a 'trademark' and that one's signature can either be tangled, illegible shapes or a series of clearly decipherable letters that distinctly spell out a person's name. However in many cases they bear no resemblance to the writer's name, but are merely identifying marks [1].

In the case of a person who is illiterate a letter $\mathrm{X}$ is used as their identifying mark and the word his/her is written above the $\mathrm{X}$ and the word mark written below.

Signature is defined as- a person's name, written in a distinctive manner; a legal form of identification or authorization [2].

Signatures are the most practiced and therefore the most automatic or habitual example of one's writing behavior. Good signatures usually exhibit one's best level of writing skills and fine motor control.

If written with a combination of speed, fluency, and deft fluctuations of pen pressure in the habitual manner of that person, a well written signature is difficult to successfully simulate.

According to Susan A. Greenfield Handwriting, especially a signature is the only universally accepted outward sign of an individual [3]. Moreover, it is a sign that is accepted as consistent.

Because signatures still play an important role in modern life, how easy do you think your signature can be forged?

Have you ever considered any of these when writing your signature?

What aspects do you think make your signature more or less easy to forge? Is your signature consistent or complex and therefore forgeryproof?

You can make your signature less vulnerable to being forged by following these suggestions: [4]
1. Develop a complex signature with numerous changes of direction that cannot be easily imitated.

2. Develop your handwriting skill until you attain a high level, which is difficult to duplicate.

3. Write rapidly.

4. Stylize your writing. The more individual the writing, the more difficult it is to imitate.

5. Be consistent in your writing. It is also advisable to have more than one style of signature, one for correspondence and one for signing financial or legal documents.

6. Illegible writing is more difficult to imitate.

\section{Complexity}

Dr. Bryan Found describes complexity (Figure 1) as the number of changes of direction of the writing and intersections [5].

\section{KNOWN}

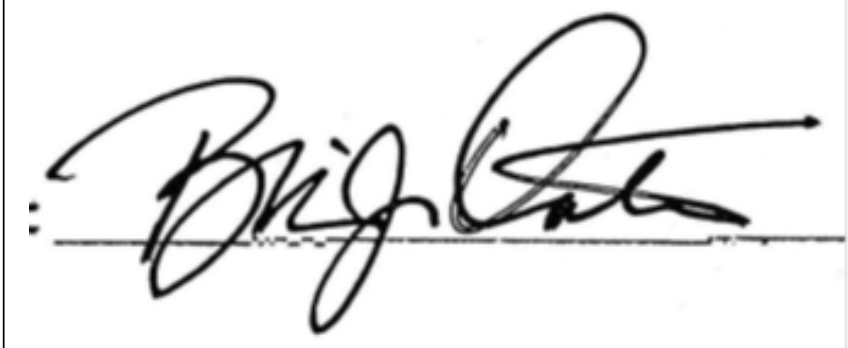

Figure 1: A complex writing contains many changes of direction and intersections, good rhythm, written rapidly, complex letter forms, high skill level.

\section{Consistency}

Consistency is the agreement among things or parts (Figures 2-4). 
Citation: Andrea Thomas-Dobson (2017) The Importance of Having a Signature that is Difficult to Imitate or Forge. J Forensic Res 8: 394 . doi:

Page 2 of 2

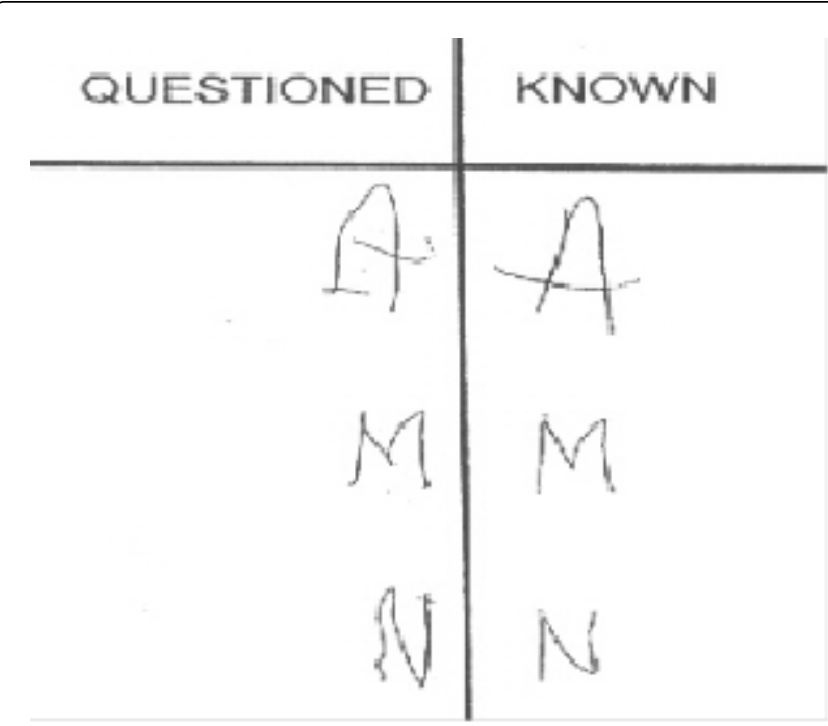

Figure 2: In relation to handwriting it is the agreement of handwriting characteristics with less variation.
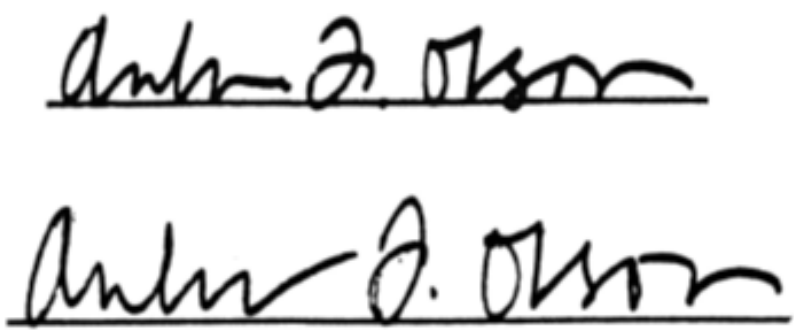

Figure 3: It is easier to identify handwriting when the writing is consistent.

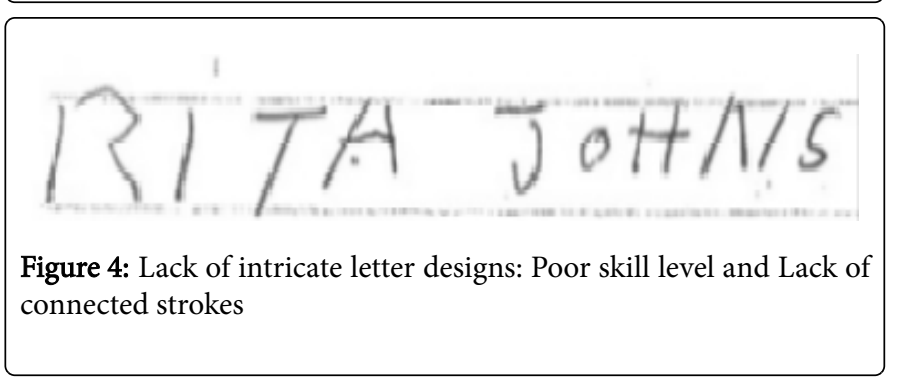

\section{Lack of Complexity}

Where a person over simplifies his or her signature it can be easily forged; that is a signature with few flourishes or change in direction of strokes. Where it is slowly written it provides forgers a better handle on imitating it as forgeries are usually drawn slowly and carefully (Figure $5)$.

To prevent yourself from falling victim to forgery, take a closer look at your signature and adopt these protective measures.

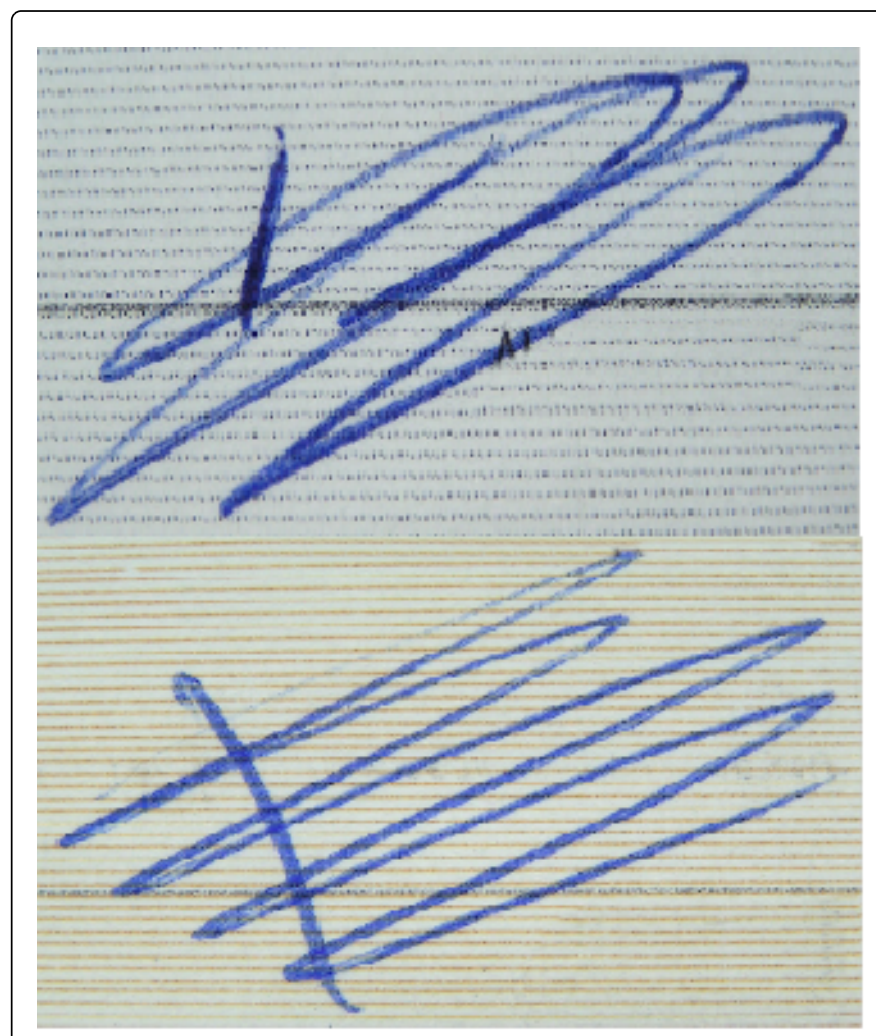

Figure 5: It is easier to create a passable forgery of versatile writers who oversimplify their writing.

\section{References}

1. Hayes R Training Course in Questioned Handwriting \& Document Examination

2. Joseph J (2010) The Layman's Glossary Relating to the Forensic Examination of Handwriting, Signatures and Documents.

3. Greenfield SA (1995) Journey to the Centers of the Mind: Toward a Science of Consciousness 1st Edition.

4. Koppenhaver KM (2002) Attorney's Guide to Document Examination; Greenwood Publishing Group.

5. http://www.latrobe.edu.au/humanbio/forensic/fepl.html 